\title{
General Material Balance for Gas Condensate Reservoir and its GIIP Estimations
}

\section{Bilal Shams ${ }^{1,2 *}$, Jun Yao' and Li Aifen ${ }^{1}$}

${ }^{1}$ Department of Reservoir Engineering, China University of Petroleum (Huadong), China

${ }^{2}$ Mehran University of Engineering and Technology (SZAB Campus-Kairpur Mirs), Pakistan

\begin{abstract}
As now-days drilling depths penetrating deeper for exploiting more reserves, hence the gaseous phase exist especially whose phase envelop lies near to critical point. From available literature, it is evident that near critical fluids are of complex phenomena due to intensive compositional change even with respect to very little change in pressure gradient. Hence for gas condensate reservoirs, especial methods for PVT analysis like Whitson and Torp K-Value method is currently used in industry to evaluate effectively the PVT properties even below the saturation pressure. On the basis of Whitson and Torp method and their available PVT equations, the material balance equation for gas condensate reservoir is required and explained in this paper as the conventional material balance is not applicable on near critical fluid reservoirs because it does not contain standard PVT properties solutions below the saturation pressure. The application of material balance equation for gas condensate reservoirs is highlighted to calculate the initial reserves and gas in-places that valuably assist in designing production development strategy and economic evaluations. Gas in-places are estimated and calculated by using application of Havlenah-Odeh plot methods and compared with conventional analysis of Pressure Decline method. Paper explanations are raised with intentions of good assistance to the petroleum industry in estimating gas condensate reserves to implementing pragmatic future development decisions.
\end{abstract}

Keywords: Gas condensate reservoir; General material balance; GIIP estimations

\section{Introduction}

Estimating the reserves and in-places, available literature concluded four methods present into the industry that are volumetric, material balance, pressure decline and reservoir simulation. As volumetric and pressure decline methods are used into early and last time period of productions respectively therefore it is not of good assistance of providing in-place estimations and different production scenarios. It is because of volumetric methods just incorporates rock properties and not PVT properties while production decline methods lead to overestimations into early production times due to ignoring rock and fluid expansion effects [1]. While reservoir simulation is a programming tool comprises of physics and thermodynamic principles in mathematical equations as a representative of reservoir rock mass and energy balance [2]. Reservoir simulation applications vary at different stages of production life depending on the available data; uncertainties lead to inaccuracies. Cumulative work-office of reservoir simulation principle is dependent of material balance solutions for fluid flow in porous media. Since material balance methods incorporate detailed PVT properties above and below the saturation pressures, hence more chances to improve to uncertainties for accurate reserve estimations.

Conventional material balance solutions like for dry and wet gas reservoirs does not incorporate standard PVT properties below the saturation pressure because it differs from condensate reservoir as their phase exist beyond the system critical temperature and cri-con-dentherm. Also the conventional material balance especially for oil saturated reservoirs based on the principle that there is no compositional change occurs below the saturation pressures of the system. Near critical fluid reservoirs especially gas condensates observed huge compositional change with respect to small change in pressure gradient, therefore especial material balance is required to estimate the reserves on available detailed PVT standard properties of the fluid system.

Walsh et al. (1994) [3,4] extended Schilthuis [5] conventional material balance equation for gas condensate reservoir contains set of standard PVT properties; equation is applicable to all ranges of reservoir fluids. This paper explains the general material balance equation for gas condensate reservoirs with detailed considerations of relevant PVT standard properties especially below the saturation pressure. The application of general material balance equation is used to estimate the gas initial in-place with assistance of effective Havlena-Odeh [6] plot method and then is compared with pressure decline analysis to identify the rock and fluid expansion effects.

\section{Material balance equation for gas condensate reservoir}

The material balance for volumetric gas condensate reservoir initializes with the mass or mole balance principle, i.e.,

$$
n_{\text {prod }}=n_{\text {initial }}-n_{\text {final }}
$$

If in terms of real gas law equation applicable production (standard), initial and final conditions: $n=\frac{p V}{z R T}$

Hence equation 1 becomes:

$$
\frac{p_{s c} \cdot\left(G_{p}+N_{p}-W_{p}\right)}{z_{s c} \cdot R \cdot T_{s c}}=\frac{p_{i} \cdot\left(G_{i}+N_{i}+W_{e}\right)}{z_{i} \cdot R \cdot T_{i}}-\frac{p_{f} \cdot\left[\left(G_{f}+N_{f}\right)-\left(W_{e}-W_{p}\right)\right]}{z_{f} \cdot R \cdot T_{f}} \rightarrow
$$

Equation 2represents saturation conditions as initial conditions

*Corresponding author: Bilal Shams, Department of Reservoir Engineering, China University of Petroleum (Huadong), China, Tel: +861663991137; E-mail memonbs@gmail.com

Receive December 07, 2015; Accepted March 22, 2016; Published March 29 2016

Citation: Shams B, Yao J, Aifen L (2016) General Material Balance for Gas Condensate Reservoir and its GIIP Estimations. J Pet Environ Biotechnol 7: 270. doi:10.4172/2157-7463.1000270

Copyright: () 2016 Shams B, et al. This is an open-access article distributed under the terms of the Creative Commons Attribution License, which permits unrestricted use, distribution, and reproduction in any medium, provided the original author and source are credited. 
including net water influx term if water aquifer is supporting volumetric condensate gas reservoir. Where $G_{p}, N_{p}$ and $W_{p}$ are produced gas, condensate and water volume from reservoir at standard condition in MScf, Stb and bbl respectively. $G_{i}$ and $N_{i}$ is the initial gas and condensate in-places reservoir volume in Mscf and Stb respectively, $G_{f}$ and $N_{f}$ are remaining gas and condensate volumes at depleted condition in Mscf and Stb respectively and $W_{e}$ is the water encroached into the reservoir. Volumes can be calculated via CVD tests producing the number of moles at each pressure depletion stage.

Estimations of critical properties yield values of physical properties. Since gas condensate reservoir flow during depletion is of complex thermodynamics yielding huge compositional change with pressure gradient change, hence the determination of physical properties from correlations needs special attention to correct the values. Whitson et al. [7] introduced Standing K-value flash method for the constant volume cell fluid test using the optimistic separator flash value at each cell depletion pressure for $n$ moles of gas and condensate production. This technique yielding approximate accurate values of physical properties and is considered very effective in the industry for condensate fluid modeling. With this technique and material balance principle, introducing respective stages physical properties by replacing their critical properties after rearranging, equation 2 becomes (assuming volume term at final conditions is zero):

$$
\begin{aligned}
& G_{\text {fgi }} \cdot E_{g w f}+N_{f o i} \cdot E_{o w f}+W_{e} \cdot B_{w}= \\
& {\left[G_{p s} \cdot \frac{\left(B_{g}-B_{o} \cdot R_{v}\right)}{\left(1-R_{v} \cdot R_{s}\right)}+N_{p s} \cdot \frac{\left(B_{g}-B_{o} \cdot R_{v}\right)}{\left(1-R_{v} \cdot R_{s}\right)}\right]-\left[\left(W_{p}-W_{i n j}\right) B_{w}\right]}
\end{aligned}
$$

Where $G_{f g i}$ and $N_{f o i}$ are formation gas initial in-place and formation condensate initial in-place in reservoir, $G_{p s}$ and $N_{p s}$ are produced gas and condensate volumes at surface and $W_{i n j}, B_{w}$ are water injection volume (in case of injection well) and water formation volume factor $(b b l / s t b)$ respectively. $B_{g}, R_{s}, R_{v}$ and $B_{o}$ are pseudo black oil PVT properties at surface termed as gas formation volume factor ( $c f$ ) $s c f)$, gas-to-condensate ratio $(s c f / s t b)$, condensate-to-gas ratio $(s t b / s c f)$ and condensate formation volume factor $(b b l / s t b)$ respectively. And $E_{\text {gwf }}, E_{\text {owf }}$ are expansion/composite factors of gas and condensate with respect to water and rock expansion and compressibility; expansion factors are defined as:

$$
\begin{array}{ll}
E_{g w f}=E_{g}+\left(\frac{B_{g i}}{1-S_{w i}}\right)\left(\frac{S_{w i} \cdot E_{w}}{B_{w i}}+E_{f}\right) & \rightarrow \\
E_{\text {owf }}=E_{o}+\left(\frac{B_{o i}}{1-S_{w i}}\right)\left(\frac{S_{w i} \cdot E_{w}}{B_{w i}}+E_{f}\right) & \rightarrow
\end{array}
$$

In equation 4 and $5, E_{g}, E_{\sigma}, E_{w}$ and $E_{f}$ are gas, condensate, water and formation expansion factors respectively that are dependent on system critical properties and composition and $S_{w i}$ is reservoir initial water saturation.

Equation 3 was proposed by Havlena-Odeh [6] on the following material balance principle expandable for the gas condensate reservoir:

Underground withdrawal $=$ Gas expansion + Water expansion $/$ Pore compaction + Water influx

Re-arranging as equation 3 is arranged, the equation 3 is:

Gas expansion (with oil condensation and water encroachentterm) $=$ Underground withdrawal - Water influx.

In equation 3, Pore Compaction term is absent as it is considered negligible with respect to gas expansion that yielding condensate oil components as pressure declines below saturation pressure.
Representing $\mathrm{F}$ as a net fluid withdrawal representative and neglecting negative sign of net water influx as water is also producing at surface along with gas condensate, i.e.,

$$
G_{p s} \cdot \frac{\left(B_{g}-B_{o} \cdot R_{v}\right)}{\left(1-R_{v} \cdot R_{s}\right)}+N_{p s} \cdot \frac{\left(B_{g}-B_{o} \cdot R_{v}\right)}{\left(1-R_{v} \cdot R_{s}\right)}+\left(W_{p}-W_{i n j}\right) B_{w}=F \quad \rightarrow
$$

Now equation 3 becomes:

$$
G_{f g i} \cdot E_{g w f}+N_{f o i} . E_{o w f}+W_{e}=F
$$

Above equation becomes if condensate-water-rock expansion term ' $E_{\text {owf }}$ ' is negligible and in case no condensation of oil occurs in the reservoir or for gas condensate reservoirs at initial (under-saturated) conditions:

$$
G_{f g i} \cdot E_{g w f}+W_{e}=F
$$

If in equation 2, volume assumed constant at initial and final conditions after producing $n_{p}$ number of moles at $n$ pressure depletion stages and preferring the initial conditions above the saturation pressure where no oil condensation occurs, also no water influx is encountering; hence equation becomes:

$$
\frac{p_{s c} \cdot G_{p}}{z_{s c} \cdot T_{s c}}=\frac{p_{i} \cdot V}{z_{i} \cdot T_{i}}-\frac{p_{f} \cdot V}{z_{f} \cdot T_{f}}
$$$$
\text { Rearranging, } \quad \frac{p}{z}=\frac{p_{i}}{z_{i}}-\left(\frac{p_{s c} \cdot T}{T_{s c} \cdot V}\right) \cdot G_{p}
$$

Where,

$$
V=43,560 A h \varnothing\left(1-S_{w i}\right) \quad(\text { in cu. ft }) \rightarrow
$$

And,

$$
G_{i}=43,560 \mathrm{Ah} \varnothing \frac{1-S_{w i}}{B_{g i}} \quad(\text { in Mscf) } \rightarrow
$$

Arranging (a) and (b): $\quad V=G_{i} . B_{g i}$

Introducing $B_{g i}$ value in (c): $\quad V=G_{i} \cdot\left(\frac{p_{s c}}{T_{s c}} \cdot \frac{z_{i} \cdot T_{i}}{p_{i}}\right)$

So with equation $\mathrm{A}$, equation 9 becomes:

$$
\frac{p}{z}=\frac{p_{i}}{z_{i}}-\left[\left(\frac{p_{i}}{z_{i}}\right) \cdot\left(\frac{1}{G_{i}}\right) \cdot G_{p}\right]
$$

Equation 8 and 10 are zero-dimensional general material balance equations in the form of Havelena-Odeh plotting method and conventional pressure decline analysis that can be used for reserve estimations of gas condensate reservoirs. These equations were proposed couple of years ago into the petroleum industry for gas and condensate reserve estimations but here the explanation with versatile dimension rose for better academics and practices.

\section{Applications of general material balance equation}

Havelena-Odeh general material balance equation for gas condensate reservoir provides valuable linear plotting method of net fluid withdrawal $(F)$ vs. gas composite factor $n_{p}$ for gas condensate inplace estimations. Also energy sources can also be confirmed either the reservoir is water-externally supported by linearly plotting $\left(F / E_{g w f}\right)$ vs. produced gas $\left(G_{p}\right)$. On $n_{p}$ number of production at each pressure depletion stage at constant volume and temperature of the system, the respective quantities of $F$ and $E_{g w f}$ can be calculated with their respective parametric values of $G_{p}, N_{p}, B_{g}, B_{o}, B_{t g}$ (two phase gas FVF), $R_{s}, R_{v}, z$ and gas equivalent quantity (produced wet gas per unit dry gas production). 
Pressure decline material balance linear plotting analysis ( $\mathrm{p} / \mathrm{z}$ vs. $\mathrm{G}_{p}$ ) also assist in accounting reservoir gas in-places but it does not account formation and connate water expansion. So it is not much effective for gas condensate reservoirs with water influx but can be used as comparison with Havelena-Odeh and energy plot methods for validating reserve estimations and reservoir external supports. All plotting scheme is the mathematical principle scheme of the formula: abscissa $=$ slope $\times$ ordinate + intercept $(y=m x+b)$.

\section{Case Study}

The proposed material balance equation for gas condensate reservoirs is applied to a case study of recently developed A-1 gas condensate well experiencing water production. The methodology followed is the comparison of proposed MB equation for gas condensate reservoir to pressure decline analytical solution for predicting gas and condensate in-places; accompanying with developing energy plot to observe the external support to the condensate reservoir. Since evident in literature that material balance solutions are not considered effective in early development phases of reservoir life comparing with pressure decline analytical solution but it can account effectively the presence of external energy source (i.e., water encroachment) to the reservoir; that pressure decline analytical solution has not this option. While this assumption might be considered for conventional and dry gas reservoirs and condensate reservoirs with water influx can be evaluated with above proposed $\mathrm{MB}$ equation at early development stage limited to PVT properties' estimation accuracy. Also detailed production data availability reduces the uncertainty and increases accuracy in reserves prediction.

The $\mathrm{X}$ gas condensate reservoir observed at initial reservoir pressure of approx. $23.4 \mathrm{MPa}$ at approximately $7350 \mathrm{ft}$ depth with pressure gradient of approx. $0.00057 \mathrm{MPa} / \mathrm{ft}$ accompanying with water aquifer at approximately $7405 \mathrm{ft}$ depth with pressure gradient of approx. $0.00311 \mathrm{MPa} / \mathrm{ft}$. At the initial pressure of reservoir, the gas compressibility is calculated $253 \times 10^{-6} \mathrm{psi}^{-1}$ while formation and water compressibility are $5 \times 10^{-6} p s i^{-1}$ and $3.18 \times 10^{-6} p s i^{-1}$, hence both can be comparable with gas compressibility to determine rock compaction and water expansion effects with gas expansion as this can determine the magnitude of condensate reservoir how much is over pressurized. Formation compressibility is estimated from Hall's correlation dependent upon formation porosity that is reported as average of $8.76 \%$ and connate water saturation is $35 \%$. Limited early production history of the A-1 well is plotted in Figure 1.
PVT simulation is performed on available fluid composition having the gas specific gravity 0.72 , molecular weight of higher fractions $(\mathrm{C} 7+)$ observed 137.7 by mole balance principle and its specific gravity calculated by PVT simulator is 0.78 . Liquid's density reported at stock tank conditions is 49.5 degrees API. Due to limited separator data and unavailability of laboratory PVT results, the PVT simulator is used to perform PVT simulations and observe CCE and CVD results. CCE results concluded that the condensate reservoir has maximum up to $0.0016 \%$ condensate saturation could occur near to the wellbore region as shown in Figure 2. Further results conclude detailed PVT properties using Whitson and Torp method that generated pseudo-black oil properties and assisted in in-places estimations by proposed $\mathrm{MB}$ solution for gas condensate reservoir and pressure decline analytical solution.

Figure 3 is the energy plot also known as Cole-Energy Plot describes the partial-linear increase in the pore volume of gas condensate reservoir due to continuous water encroachment into it. As the plot's slope line is not parallel to $\mathrm{x}$-axis hence it represents the presence of water aquifer attached to gas condensate reservoir. While the magnitude is concluded as weak/partial water drive as the data points have slight upward concave curvature in between 0.6 to $1 \mathrm{MMSCF}$ Gp and are not following exactly on the slope line. Cole concluded from his research that data points if have slight upward concave curvature in an energy plot represents a reservoir has partial water drive mechanism.

Figure 4 is conventional pressure decline analytical scheme solution describing the presence of external support (water aquifer) to the gas condensate reservoir as the data points are not following onto slope line while data points in between 0.8 to 1.3 MMSCF Gp experiencing downward concave curvature which explains the partial/weak water drive mechanism to the condensate reservoir. The wet gas in-place estimated from this solution is 7.8 MMSCF while it cannot has option to predict water encroachment volume.

Figure 5 is based on the results concluded from proposed material balance solution for gas condensate reservoir and plotted in a manner of produced gas and condensate volume vs. total expansion factor of gas condensate reservoir. This technique was proposed by Havlena and Odeh while concluding their material balance estimation results. By

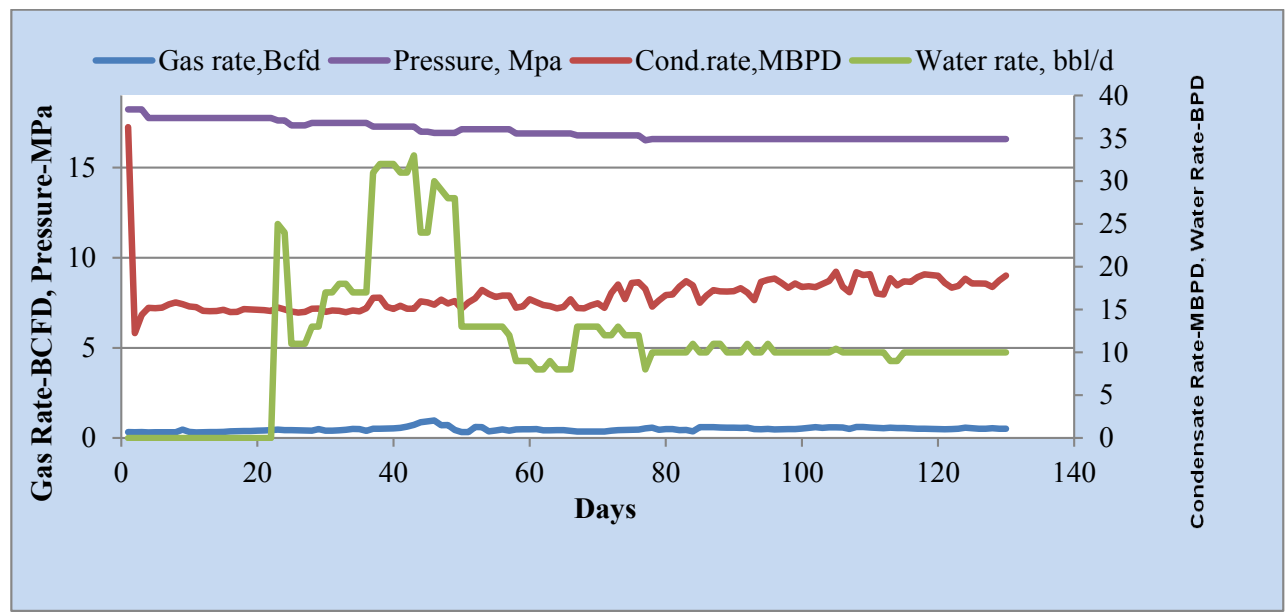

Figure 1: Pressure production history of A-1 Gas condensate well. 
Citation: Shams B, Yao J, Aifen L (2016) General Material Balance for Gas Condensate Reservoir and its GIIP Estimations. J Pet Environ Biotechnol 7: 270. doi:10.4172/2157-7463.1000270
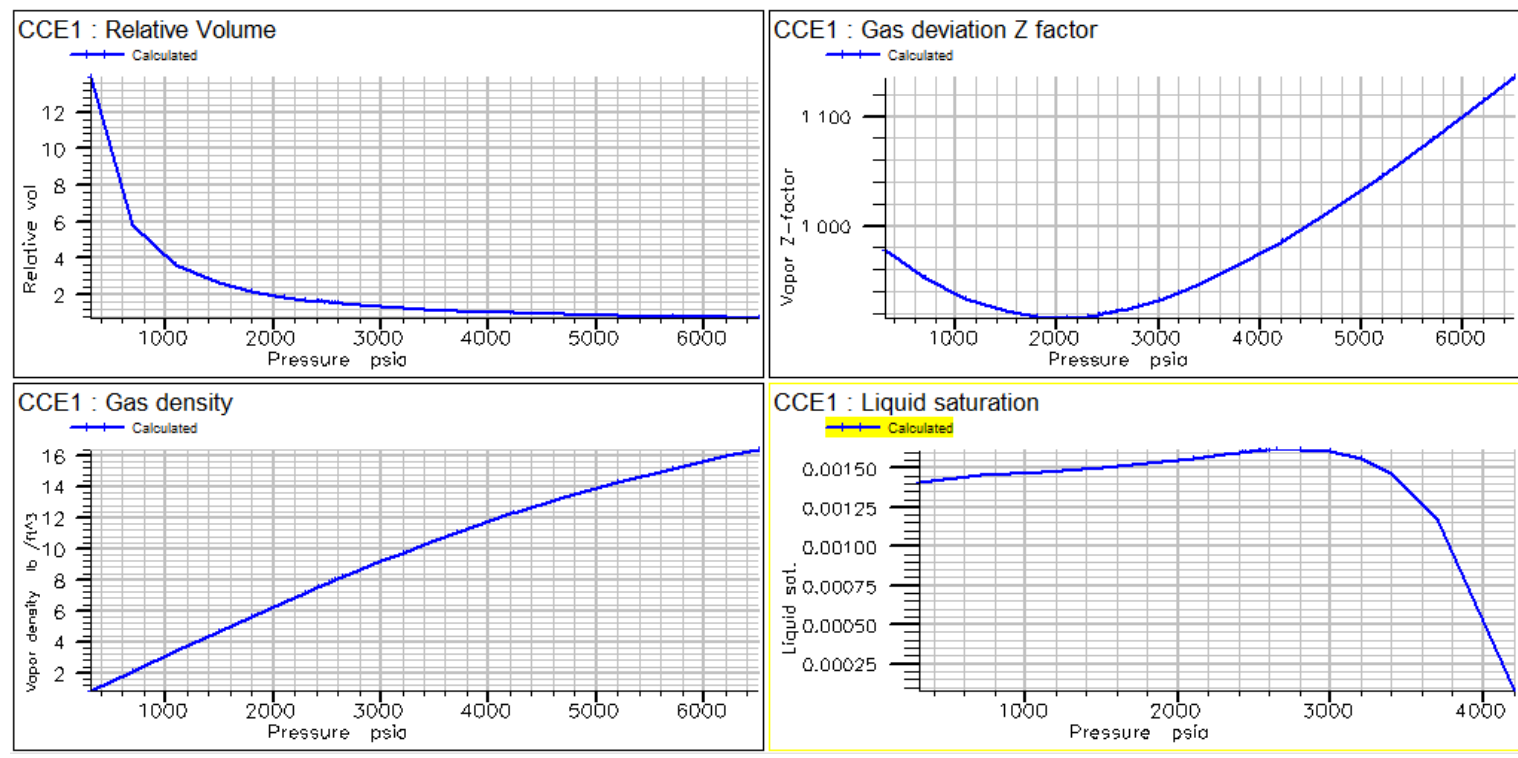

Figure 2: CCE results - Bottom right shows Condensate saturation vs. Pressure results.

F/Et vs Gp Plot

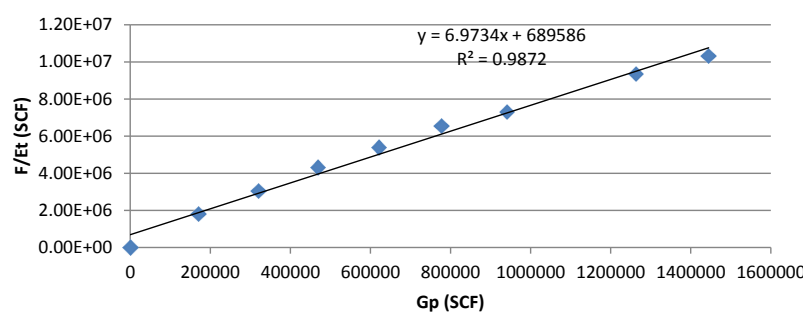

Figure 3: Energy plot of A-1 Gas condensate well.

\section{P/z vs Gp Plot}

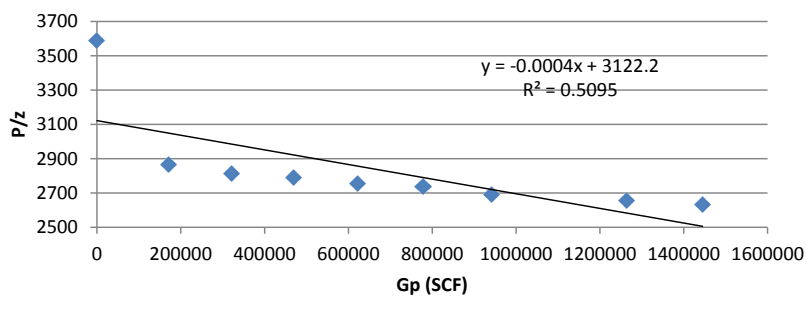

Figure 4: Pressure decline analytical Plot of A-1 Gas condensate well.

using this effective technique, the wet gas condensate in-place estimated is about 10MMSCF while water influx volume is $0.41 \mathrm{MMBbls}$. Since the slope line does not containing any data points and $\mathrm{R}^{2}$ is not equal to 1 (i.e., $65.58 \%$ is the wet gas saturation to that gas condensate reservoir), hence plot is evidently concluding the presence of water influx into the reservoir.

Comparing the results of (Figures 4 and 5), there is difference of $22 \%$ between their results. Detailed results are concluded in the following (Table 1).

\section{F vs Et Plot}

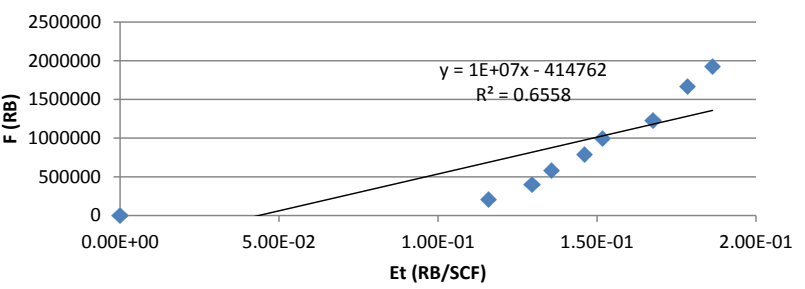

Figure 5: Proposed MB solution Plot of A-1 Gas condensate well.

\begin{tabular}{|l|c|c|c|}
\hline Methods & $\begin{array}{c}\text { Wet Gas In-place } \\
\text { (MMSCF) }\end{array}$ & $\begin{array}{c}\text { Dry Gas In-place } \\
\text { (MMSCF) }\end{array}$ & $\begin{array}{c}\text { Condensate In-place } \\
\text { (MSTB) }\end{array}$ \\
\hline $\begin{array}{l}\text { Pressure Decline } \\
\text { Analytical Solution }\end{array}$ & 7.805 & 7.786 & 2.47 \\
\hline MB Solution & 10 & 9.975 & 3.17 \\
\hline
\end{tabular}

Table 1: In-places estimation results of A-1 Gas condensate well.

\section{Conclusion}

Adopting the plotting approach of Havlena-Odeh and Cole to conclude the proposed MB solution results of A-1 Gas condensate well, it is evident the presence of water aquifer attached with the reservoir. While detailed production and separator data will give accurate results about the aquifer size. Since the reservoir is at its earlier development stage hence pressure decline analytical results could be considered valid for in-places estimation but it does not account formation and connate water expansion effects, hence proposed material balance solution for gas condensate reservoir must be considered for comparison of inplaces estimations limited to accuracy in PVT properties calculations.

\section{References}

1. Sidiqui F, Waqas GM, Khan MN (2010) Application of general material balance on gas condensate reservoirs GIIP estimations. SPE-142847, SPE/AAPG Annual Technical Conference-Islamabad, Pakistan. 
Citation: Shams B, Yao J, Aifen L (2016) General Material Balance for Gas Condensate Reservoir and its GIIP Estimations. J Pet Environ Biotechnol 7: 270. doi:10.4172/2157-7463.1000270

Page 5 of 5

2. "Reservoir Simulation" by Heriot-Watt University.

3. Walsh MP (1994) New improved equation solves for volatile oil and condensate reserves. Oil and Gas Journal.

4. Walsh MP, Towler BF (1995) Method computes PVT properties for gas condensates. Oil and Gas Journal.
5. Schilthuis RJ (1936) Active oil and reservoir energy. Trans. AIME 118: 33-52.

6. Havlena D, Odeh AS (1963) The material balance equation as an equation of straight line. Journal of Petroleum Technology.

7. Curtis HW, Stein BT (1983) Evaluating constant-volume depletion data. Journal of Petroleum Technology. 